\title{
A REPORT ON THE USE OF CHLOROQUINE SULPHATE IN LEPRA REACTION
}

\author{
K. Ramanujam, m.B.B.s. \\ Medical Officier, Government Silver Jubilee Children's Clinic, \\ Saidapet, Madras, India
}

\section{Introduction}

Chloroquine sulphate, the latest and widely used synthetic anti-malarial, had shown in recent years greater spheres of its usefulness in the treatment of amoebiasis, taeniasis, rheumatoid arthritis and lupus erythematosus. The possible therapeutic usefulness of this drug in lepra reaction was first reported from a hospital for tropical diseases in Paris, where a woman leprosy patient in the grip of lepra reaction was thought to be suffering from malaria, and treated with chloroquine. The presence of malarial infection was disproved, but it was observed that there was a rapid amelioration of the symptoms of reaction on chloroquine. This observation was followed up and reported on favourably by other workers. ThangaraJ ${ }^{1}$ using Camaquin brand of amodiaquin hydrochloride orally in a group of 11 lepromatous cases compared the result with two other groups of patients, one group of 13 cases treated with intravenous injection of potassium antimony tartrate, and another group of 14 cases as controls. As a result of this investigation he came to the conclusion that "Camoquin is effective in the control of acute lepra reaction in lepromatous leprosy" and that "it achieves its effect in less time than potassium antimony tartrate".

Another interesting observation about the usefulness of chloroquine came from MERKLEN and RIOU ${ }^{2}$ who had reported that with concurrent administration of chloroquine, the reaction could be controlled without interruption of sulphone therapy. They added that combination of chloroquine and DDS was beneficial in bringing up the dose of DDS and in two instances chloroquine could be substituted for d-cortisone. They recommend a dose of $600 \mathrm{mgm}$. initially and then a maintenance dose of 300-400 mgm.; they considered that doses less than $200 \mathrm{mgm}$. a day were ineffective.

$\mathrm{RAMU}^{3}$ reported symptomatic relief in all the 8 cases of lepra reaction he treated with chloroquine diphosphate. His dosage regimen was one tablet (equal to $250 \mathrm{mgm}$. of the base) three times a day for one week and later one tablet twice a day for another 2 weeks. The time taken for relief to set in under this regimen varied from 4 days to 14 days. He came to the tentative conclusion that chloroquine is a useful drug in the treatment of lepra reaction.

\section{Object of the Study}

Barring the oral administration of corticosteroids, the only effective treatment of acute lepra reaction has been the parenteral 
administration of antimony preparations. With the extension of mass anti-leprosy treatment in endemic areas, more cases of leprosy come under medical surveillance and more cases of lepra reaction are bound to be detected. The accepted line of treatment for lepra reaction, namely the parenteral administration of antimony, offers certain practical difficulties in rural areas. Therefore the favourable reports on the effectiveness of orally administered antimalarial drugs in the control of lepra reaction have prompted this investigation.

The object of the investigation is two-fold: (1) To find out how effectively and in what period of time chloroquine sulphate is able to control lepra reaction and, (2) how far this drug will be useful in controlling the reactions which occur very frequently in a small number of cases, and so render almost impossible the institution of adequate anti-leprosy treatment.

\section{Material for Study}

Between March and September 1959, 27 cases of lepromatous leprosy who reported with lepra reaction of varying grades of severity were accepted for the investigation. This group included 2 cases who were almost in a state of perpetual reaction, which made them incapable of taking the leprosy treatment.

\section{The Method}

The cases for investigation were entered on a proforma along with the details of the patient's condition - such as the drug he was on, whether it was the first reaction or one of several; and also the symptoms and signs such as fever, joint pain, nerve pain, bodily pain, presence of rose-spot nodules, subcutaneous nodules, exacerbation of existing lesions, onset of new lesions, turgescence of the nasal mucous membrane and the condition of the ocular elements. Details of treatment given and the progress of the case from time to time was recorded.

"Nivaquine" brand of chloroquine sulphate was administered orally in doses of $400 \mathrm{mgm}$. twice a day, equivalent to $300 \mathrm{mgm}$. of chloroquine base. This dose was maintained till the symptoms showed a tendency to abate, and then it was tailed off to $200 \mathrm{mgm}$. twice a day and later on to $200 \mathrm{mgm}$. a day. The drug was discontinued once the symptoms and signs of reaction completely disappeared. In some instances, where "experienced" patients asked for the potassium antimony tartrate injections, chloroquine was given orally along with intravenous injections of $2 \mathrm{ml}$. of pyrogen-free sterile distilled water. This clinic being only an outpatient department, no four-hourly temperature charts were maintained; the patients carried away two days' supply of the drug at a time and when they reported again, an examination as to the patients' condition was made and an enquiry was also made with regard to any untoward 
symptoms. As far as possible no leading questions with regard to the toxic symptoms due to chloroquine were put to the patient, but in a few instances it was necessary, because some of the patients as a matter of course attributed any adverse symptoms to the chloroquine. In a few instances, the patients discontinued tablets after taking them for 2-4 days. However, when they turned up again, their condition was noted although the chloroquine tablets had been discontinued in the meantime.

\section{Results}

For purposes of analysis of the results, the group of 27 cases of lepra reaction have to be split into two sub-groups-one of the 25 cases who had pure and simple lepra reaction and a small group of 2 cases who were in a state of almost continuous lepra reaction. The results obtained in these cases have been graded into three categories - "Relieved", "Improved" and "Failed". By "Relieved" it is meant cases where there is total relief from symptoms and signs of reaction; in the "Improved" cases although there was considerable relief, some remnant of the reaction syndrome such as mild neuritis or arthritis still persisted.

1. In Acute Lepra Reaction: Out of the 25 cases treated with Nivaquine brand chloroquine sulphate, 4 cases discontinued treatment for no special reason. Out of the balance of 21 cases, nine showed complete relief, $(42.86 \%)$ and twelve showed improvement $(57.14 \%)$.

The duration of treatment varied from 2 to 14 days. In 3 instances the patients discontinued treatment after 2-4 days chloroquine sulphate therapy; but when they were seen again a week to 10 days after discontinuance, they showed considerable improvement. On enquiry the patients said that they did not ask for the tablets again because they had considerably improved. The average period taken for the control of reaction was 7 days.

2. In Chronic Lepra Reaction: Two cases of lepromatous leprosy who had been on oral DDS therapy for a considerable period with good results entered a state of frequent lepra reaction-a relentless recurrence of the reaction, whether any specific anti-leprosy treatment was employed or not. In one case (case No. 3) the reaction used to be quite severe and in the other case (case No. 14), apart from slight exacerbation of lesions and E.N.L. phenomenon, nerve pain was quite agonising. Both these cases were given chloroquine sulphate, beginning with $400 \mathrm{mgm}$. twice a day and then tailing off to $200 \mathrm{mgm}$. a day. In the first case where the chloroquine sulphate administration lasted 11 weeks, it was possible to administer DDS also orally to a maximum of $50 \mathrm{mgm}$. per day, without any untoward sign or symptom. At the time when the patient was really getting stabilised on DDS oral therapy under chloroquine protection, he discontinued. 
In the second case (case No. 14) one of almost continuous reaction, the usual measures failed to control the reaction. He was put on chloroquine $200 \mathrm{mgm}$. twice a day and after 5 days there was complete subsidence of pain, although E.N.L. spots continued to occur. The dose was kept up at the same level and drug continued for a period of 7 weeks, because the reaction signs and symptoms showed a tendency to recur. At the end of this period, the patient said he was feeling "exhausted" and hence chloroquine was discontinued. After a break of 5 weeks, the reaction symptoms recurred and the patient was once more put back on chloroquine $200 \mathrm{mgm}$. b.d. After 5 weeks' treatment in the second course, there was considerable relief and then the patient was started on small doses of DDS orally, keeping him on a maintenance dose of $200 \mathrm{mgm}$. chloroquine per day. To this date, the patient is continuing sulphone in small doses, without the recurrence of lepra reaction. The E.N.L. spots keep coming in smaller numbers, and there is freedom from the agonising neuritis and arthritis.

3. In Recurrent Reaction: In 5 cases, chloroquine sulphate was administered on more than one occasion, when the reactions recurred - Case No. 1 had three courses, Case No. 2 two, Case No. 8 four courses and Case No. 9 two and Case No. 17 two. In cases Nos. 2 and 9 , the results were good on both the occasions. In cases Nos. 1 and 8 , the drug failed on one occasion in each and here relief was obtained by the intravenous injection of potassium antimony tartrate. In case No. 17, during the second course of treatment with chloroquine, the patient developed troublesome vomiting and hence the drug was discontinued.

\section{Toxicity of Chloroquine}

Toxic reactions attributable to the drug were encountered in 7 cases. In the order of frequency they were, vomiting in 4 cases, nausea in 1 case, transitory giddiness in one and psychosis in 1 case. In only one instance, vomiting was so severe as to necessitate withdrawal of the drug. In the rest, the vomiting was controlled either by withholding the drug for a day or two or with the administration of "Avomine".

One case (case 22) manifested signs of drug psychosis towards the end of chloroquine therapy. The patient had almost completely recovered from the reaction but exhibited unusual behaviour-he became apprehensive and disoriented: chloroquine was withdrawn and patient put on "Nevrovitamine 4-adults" and the symptoms passed off after about 10 days.

The general impression was that the toxic manifestations were of a mild nature, easily controllable. 


\section{General Comments}

The mode of action of chloroquine in the lepra reaction syndrome is not clearly understood. PESTEL who considered lepra reaction a nonspecific inflammatory process attributed the beneficial effect of chloroquine in this condition to its anti-inflammatory properties. MERKLEN contended that lepra reactions are not purely inflammatory since he had observed "the occurrence of the typical nodules of the disease". However, he confirmed the usefulness of Camoquin in this condition and considered it to be "more effective than K.thrombyl and less dangerous than phenyl-butazone".

Whatever may be the mode of action of chloroquine in lepra reaction the drug seems to have a definite place in the treatment of this condition. Although concurrent trials with potassium antimony tartrate have not been undertaken side by side with that of chloroquine, it can be said without reservation that the intravenous antimony therapy is quicker by far and more complete in controlling acute lepra reaction. This opinion is expressed from the experience gathered in the use of this preparation for the past several years. However, the great advantage with chloroquine sulphate is its easy administration, namely oral, which makes it very handy in the treatment of this complication in rural areas.

Something more important than just the treatment of acute lepra reaction with chloroquine is the assessment of the value of the drug in the treatment of recurrent, subacute, or chronic lepra reactions which make it impossible for the patient to catch up with his disease with adequate anti-leprosy treatment. Corticosteroids have been widely acclaimed as the drug of choice under these conditions. But the prohibitive cost of the drug and the none too favourable economic status of the leprosy patients necessitate the search for a cheaper, effective drug. Could this be chloroquine? From the results obtained in two cases it would be unwise to assess the efficiency of chloroquine in prolonged lepra reaction. However, the available evidence, though meagre, indicates its possible usefulness in the treatment of this condition. This deserves further investigation.

\section{Summary}

The usefulness of chloroquine sulphate in the treatment of lepra reaction is reported. The results indicate that the drug is effective in controlling acute lepra reaction arising in lepromatous leprosy. The easy administration of the drug by the oral route enhances its value as a therapeutic agent that is capable of wide application. Chloroquine seems to hold out a possibliity of controlling the "smouldering" lepra reaction and making the induction of sulphone therapy possible. 


\section{Acknowledgements}

Thanks are expressed to the patients for their willing co-operation in this investigation; to Messrs. May \& Baker (India) Private Ltd., Madras, for their generous supply of Nivaquine brand of chloroquine sulphate; to the Director, Central Leprosy Teaching \& Research Institute, Chingleput for permission to publish this paper.

\section{References}

I. Thangaraj, R. H., Leprosy Review, 30, 2, 1959, p. 106.

2. Merklen, F. P. and Riou, M. R., Presse Medicale, 66, 43, p. 984.

3. Ramu, G., Journal of the Indian Medical Association, 33 4, p. 127. 